\title{
Experimental Validation of a Forward Looking Interferometer for Detection of Clear Air Turbulence due to Mountain Waves
}

\author{
Philip R. Schaffner ${ }^{1}$ and Taumi S. Daniels ${ }^{2}$ \\ NASA Langley Research Center, Hampton, Virginia 23681 \\ Leanne L. West ${ }^{3}$, Gary G. Gimmestad ${ }^{4}$ Sarah E. Lane ${ }^{5}$, and Edward M. Burdette ${ }^{6}$ \\ Georgia Tech Research Institute, Atlanta, Georgia 30332 \\ William L. Smith ${ }^{7}$ and Stanislav Kireev ${ }^{8}$ \\ Hampton University, Hampton, Virginia 23668 \\ Larry Cornman ${ }^{9}$ and Robert D. Sharman ${ }^{10}$ \\ Science and Technology in Atmospheric Research [STAR] Institute, Boulder, Colorado 80301
}

\begin{abstract}
The Forward-Looking Interferometer (FLI) is an airborne sensor concept for detection and estimation of potential atmospheric hazards to aircraft. To be commercially viable such a sensor should address multiple hazards to justify the costs of development, certification, installation, training, and maintenance. The FLI concept is based on high-resolution infrared Fourier Transform Spectrometry (FTS) technologies that have been developed for satellite remote sensing. These technologies have also been applied to the detection of aerosols and gases for other purposes. The FLI is being evaluated for its potential to address multiple hazards, during all phases of flight, including clear air turbulence (CAT), volcanic ash, wake vortices, low slant range visibility, dry wind shear, and icing. In addition, the FLI is being evaluated for its potential to detect hazardous runway conditions during landing, such as wet or icy asphalt or concrete. The validation of model-based instrument and hazard simulation results is accomplished by comparing predicted performance against empirical data. Models for FLI measurables for mountain wave turbulence were developed during the previous phases of the project. Prior to the field campaign, these models were used to predict what the sensors should have been able to detect, based on expected instrument performance. After the data collection activities, the empirical data was used to update and validate the existing models. This iterative process was employed during the course of the project as new empirical results became available. Previous research programs, focused on forward-looking airborne sensors such as Doppler radars and lidars to detect and forecast turbulence, have produced many tools for analysis, modeling, and simulation. Following on the methods used in the airborne radar turbulence detection problem, relationships between the statistics of an atmospheric disturbance (such as the temperature field) and those of the
\end{abstract}

Aerospace Technologist, Research Directorate Electromagnetics \& Sensors Branch, 8 N Dryden Street, MS 473.

Aerospace Technologist, Research Directorate Electromagnetics \& Sensors Branch, 8 N Dryden Street, MS 473.

Principal Research Scientist and Director, Landmarc Research Center, Electro-Optical Systems Laboratory, 925

Dalney St., MS 0834.

Principal Research Scientist, Electro-Optical Systems Laboratory, 925 Dalney St., MS 0834, AIAA Member.

Research Scientist I, Electro-Optical Systems Laboratory, 925 Dalney St., MS 0841.

Research Scientist I, Electro-Optical Systems Laboratory, 925 Dalney St., MS 0841.

Distinguished Professor, Dept. of Atmospheric and Planetary Sciences, 21 Tyler Street.

Research Assistant Professor, Dept. of Atmospheric and Planetary Sciences, 21 Tyler Street.

Project Scientist, Research Applications Laboratory, Aviation Applications Program, Senior Member AIAA .

Project Scientist, Research Applications Laboratory. 
sensor measurements (the spectral radiance) will be developed. In the mountain lee wave data collected in the previous FLI project, the data showed a damped, periodic mountain wave structure. The wave data itself will be of use in forecast and nowcast turbulence products such as the Graphical Turbulence Guidance (GTG) and Graphical Turbulence Guidance Nowcast (GTG-N) products. Determining how turbulence hazard estimates can be derived from FLI measurements will require further investigation.

\section{Introduction}

$\mathrm{T}$ HE Forward-Looking Interferometer (FLI) is an airborne sensor concept for detection and estimation of potential atmospheric hazards to aircraft. To be commercially viable such a sensor should address multiple hazards to justify the costs of development, certification, installation, training, and maintenance. The FLI concept is based on high-resolution infrared Fourier Transform Spectrometry (FTS) technologies that have been developed for satellite remote sensing. These technologies have also been applied to the detection of aerosols and gases for other purposes. The FLI is being evaluated for its potential to address multiple hazards, during all phases of flight, including clear air turbulence (CAT), volcanic ash, wake vortices, low slant range visibility, dry wind shear, and icing. In addition, the FLI is being evaluated for its potential to detect hazardous runway conditions during landing, such as wet or icy asphalt or concrete.

Prior research has addressed the physical basis for radiometric detection of these hazards. ${ }^{1-3}$ The ability of the FLI to provide estimates of the range to in-flight hazards was also investigated, with the result that both detection and ranging are enabled by the high spectral resolution provided by the FTS. A sufficient infrared spectral signature appeared to be associated with the hazards to enable detection and/or mitigation of all of them, although detection of CAT at relevant ranges may also require that the FLI have high temperature resolution, dependent on a good signalto-noise ratio. The combination of high spectral and temperature resolutions in an imaging instrument is expected to enable sophisticated algorithms with high detection rates and low false alarm rates. The FLI will also function as an infrared imager, providing a real-time video display with night vision capability and enhanced vision in obscured conditions. These capabilities will enable the FLI to detect runway obstructions during landing, including both vehicles and wild game or livestock.

The current project includes several ground-based field data collection events for hazards, including runway surface state and contamination, low visibility conditions, wake vortices, and mountain wave turbulence. The research is currently focused on mountain wave turbulence, and field data collection was conducted in November 2011 at the University of Colorado-Boulder's Mountain Research Station (MRS) near Boulder, CO. The Mountain Research Station (MRS) is located on the front range of the Rocky Mountains at an elevation of 2,900 meters $(9,500$ feet), approximately 76 kilometers (47 miles) west northwest of Denver International Airport (DEN). This elevation and clear line of sight to the atmosphere above the airport make MRS an ideal location for observing mountain waves that form over the Rocky Mountains. The instruments were located looking out of an open window from the second floor of the MRS dining hall, which provided a clear view of the sky over the trees in the direction of Denver, as shown in Figure 1. 

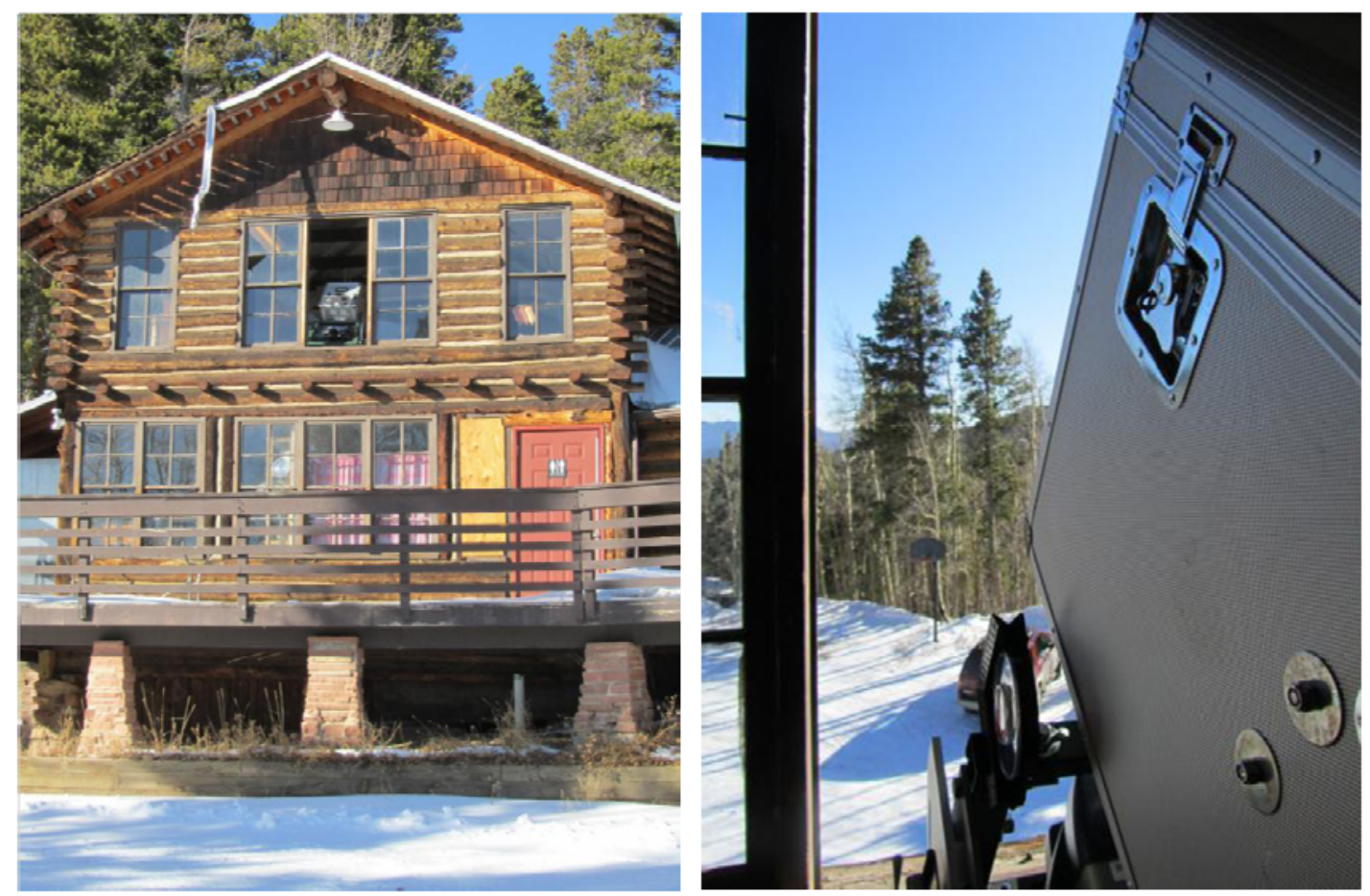

Figure 1. MRS dining hall and instrumentation

The mountain wave research experiment has three major elements: 1) ground-based field measurements; 2) the validation of models and simulations by comparing predicted results to empirical data; and 3) the development of improved sensor models and hazard metrics. Simulations to determine test requirements Weather Research and Forecast (WRF) model data - temperature and $\mathrm{H}_{2} \mathrm{O}$ profiles) were generated from an actual lee wave event on March 6,2004 . Although these fields do not develop identically for all cases, the model is perhaps representative of what one could expect of mountain waves in general. The vertical cross-section of the water vapor perturbations for this representative case is shown in Figure 2.

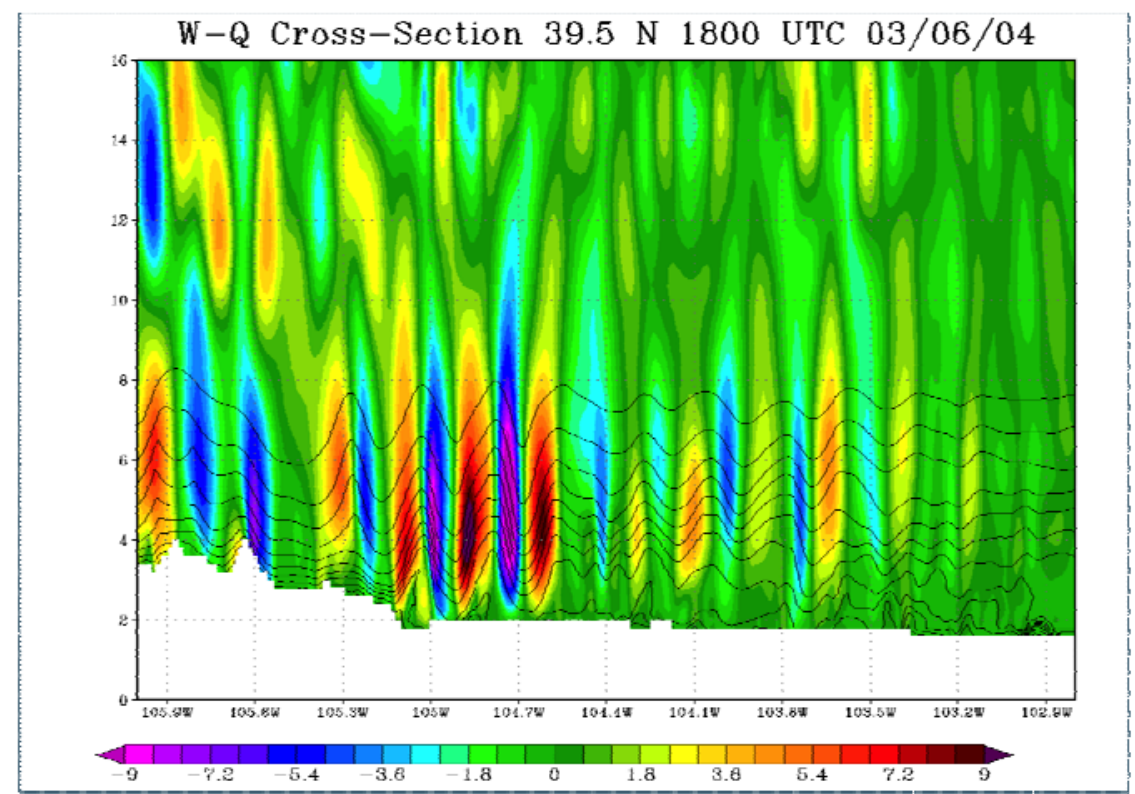

Figure 2. Vertical cross-section of water vapor for lee wave turbulent event, March 6, 2004. 
In these simulations, the observer was placed at the Hyper-Cam location at MRS and directed east at 15, 30, 45, 60 , and $90^{\circ}$ elevation angles. The brightness temperature differences from the azimuthal mean from the simulation that most closely match the actual data collection configuration at MRS are shown in Figure 3; note that although the color scale was limited to $+/-0.5 \mathrm{~K}$ in order to accentuate signals beyond these limits, the results from the simulations in general did indicate that signals of 1 Kelvin or more should be observable. Distance to turbulence should be predictable from the spectrum due to differences in absorption along the sides of the spectral window, though this capability is only possible if the data is collected at $1 \mathrm{~cm}^{-1}$ resolution. The data collected at MRS in November was collected at $1 \mathrm{~cm}^{-1}, 4 \mathrm{~cm}^{-1}$ and $16 \mathrm{~cm}^{-1}$ resolutions. The $1 \mathrm{~cm}^{-1}$ datasets have not yet been investigated.

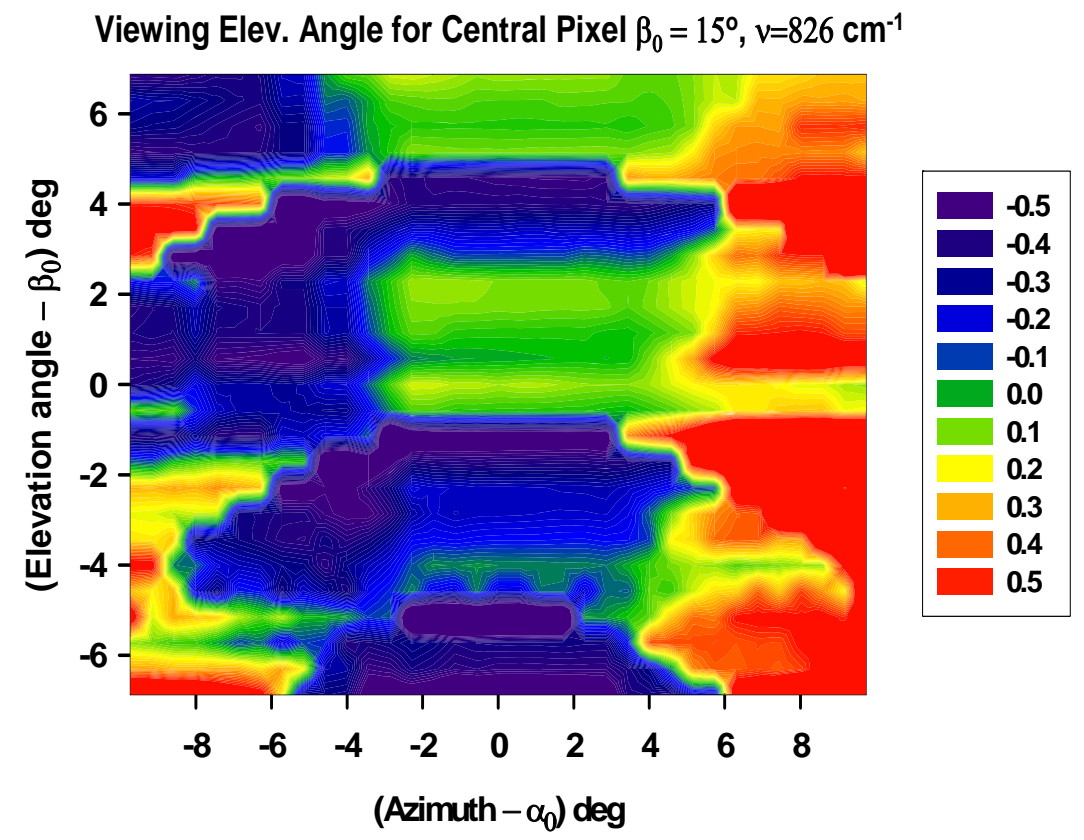

Figure 3. Brightness temperature difference (Kelvin) from the azimuthal mean.

\section{Experimental Instrumentation}

Instrumentation for these field tests included both a Telops Long Wave InfraRed (LWIR) Hyper-Cam interferometric imager and a Designs and Prototypes (D\&P) TurboFT single pixel spectro-radiometer. Simulations have shown that mountain waves will cause a pattern of brightness temperature differences that should be observable with both the Hyper-Cam and the D\&P. The Hyper-Cam combines imaging with high spectral resolution in the $870-1300 \mathrm{~cm}^{-1}$ infrared window region, but it requires times of seconds to minutes to acquire a single data cube, depending on the resolution and number of pixels used. These acquisition times are appropriate for mountain waves, which are almost stationary. These measurements were taken at the MRS, located at an altitude of 9,500 feet, so that measurements were gathered in a forward-looking manner, per the diagram in Figure 4 below. 


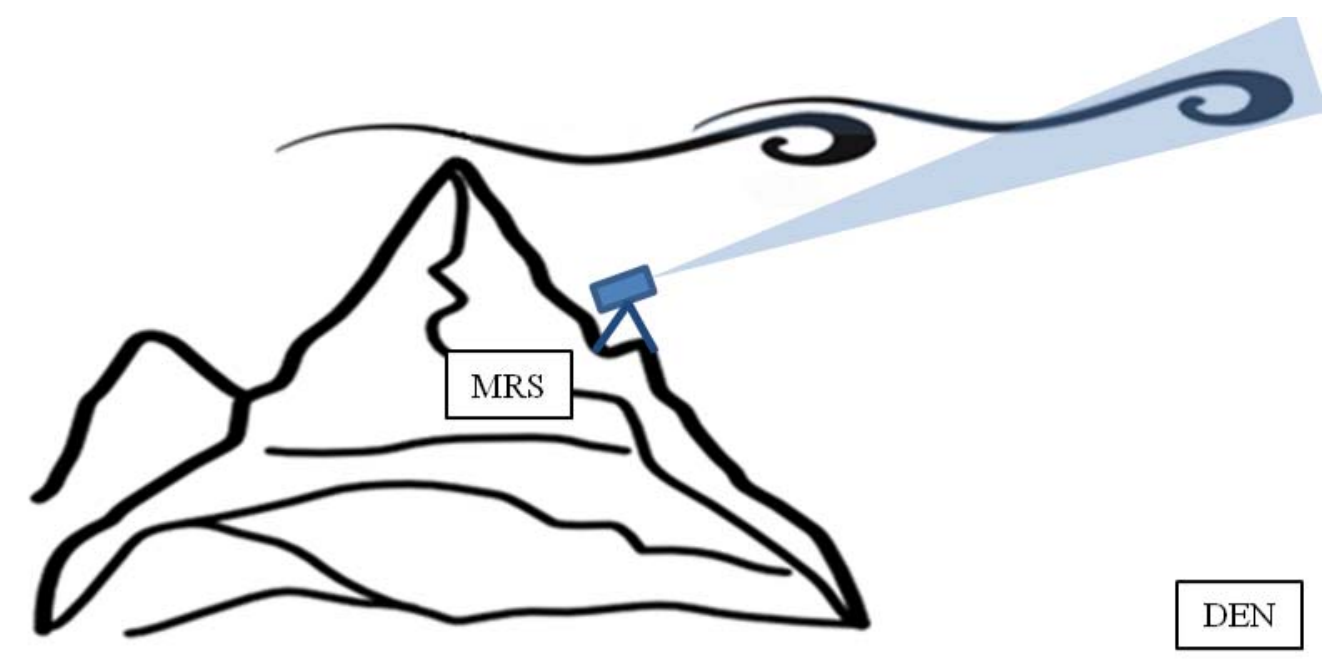

Figure 4. Mountain Wave Data Collection

The D\&P spectrometer is complementary to the Telops instrument: it is very fast, with a set resolution of $4 \mathrm{~cm}^{-1}$ and a wide spectral range of $625-5000 \mathrm{~cm}^{-1}$, with a single line of sight. In a previous phase of the program, data was collected at the NCAR Foothills Lab in Boulder, CO, in 2008. That data consists of sets of 5,000 spectra recorded at three per second, and statistical analyses of those time series have revealed fluctuations in the atmospheric spectral absorption window regions that are different on windy days and calm days. The D\&P is expected to enable detection of fast fluctuations associated with turbulent air motion. In addition, its extended spectral range enables further analysis in the mid-wave infrared (MWIR) region of the spectrum.

\section{Ancillary Data Collection}

Other data collected includes weather and satellite data. Information such as winds, temperature, humidity, visibility, etc. will be valuable for later modeling and is important to understanding the sensor data collected. Atmospheric data was provided by the MRS on-site weather station and the Science and Technology in Atmospheric Research (STAR) Institute located about 45 minutes away in Boulder, CO. The location of the tests, on the front range of the Rocky Mountains, was advantageous for acquiring truth data due to the amount of air traffic in the area primarily comprising flights into, out of, and at cruise altitudes over the Denver International Airport (DEN). Air traffic provided a source for pilot reports (PIREPS) and automated reports from United Airlines (UAL) 757 and Delta Airlines (DAL) 737 aircraft as "ground truth" for the turbulence detection efforts.

The UAL and DAL aircraft run algorithms to estimate and automatically report eddy dissipation rates (EDR) to the one-third power. These reports consist of the peak and mean value of the EDR over the previous minute (in cruise). These reports cover "none" to "extreme" levels of turbulence. The truth data allow for the evaluation of both true detections and false alarms by the sensor and algorithms. Pilot reports (PIREPS) are subjective in nature. PIREPS are not always given for turbulence encounters (even severe ones); they are rarely given for null turbulence; the reported time and position can be in error; and the intensity level of the turbulence reported by the pilot is a subjective measure of the actual intensity level. Nevertheless, these data, when available, are valuable in the verification process. Figure 5 shows the flight tracks of the EDR-equipped UAL 757s over a 24-hour period. It can be seen that there are many flights in the Colorado area. 


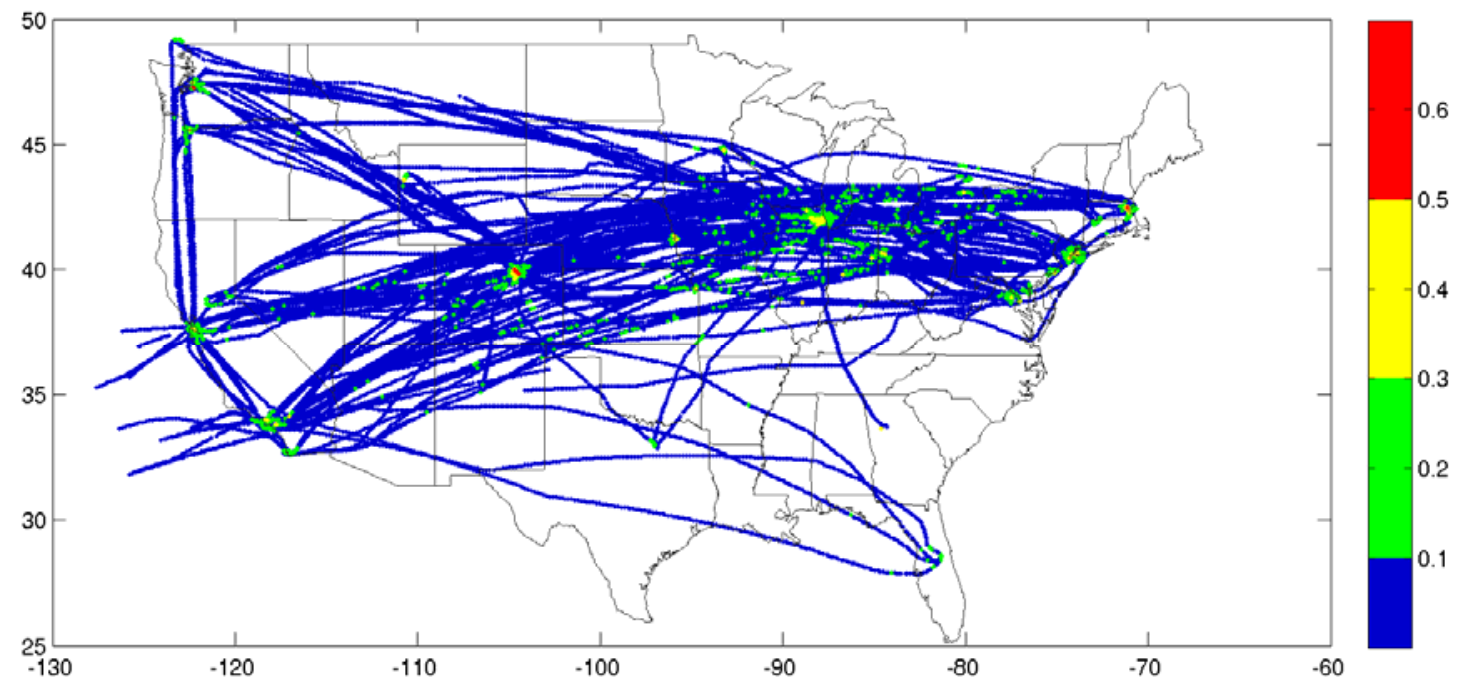

Figure 5. UAL 757 reports over a 24-hour period. Colors are assigned based on severity of turbulence. $(\mathbf{0}=$ None $/$ Smooth; $0.1=$ Smooth to Light; $0.2=$ Light; $0.3=$ Light to Moderate; $0.4=$ Moderate; $0.5=$ Moderate to Severe; $0.6=$ Severe; $0.7=$ Severe to Extreme)

A useful tool for monitoring turbulence activity during the field test was the "EDR viewer." This viewer allows for the display of real-time and archival EDR data sets, which are displayed as color-coded square dots. Furthermore, the EDR viewer contains turbulence forecast information from the Graphical Turbulence Guidance (GTG) forecast product. The GTG data was used in real time during a data collection period and in archival mode for the verification effort. The viewer can also generate vertical cross-sections.

Additionally, MODIS satellite imagery was viewed during the test to identify days with mountain waves. A MODIS image from November 17, 2011 is shown in Figure 6. This figure clearly shows evidence of mountain waves along the Rocky Mountains.

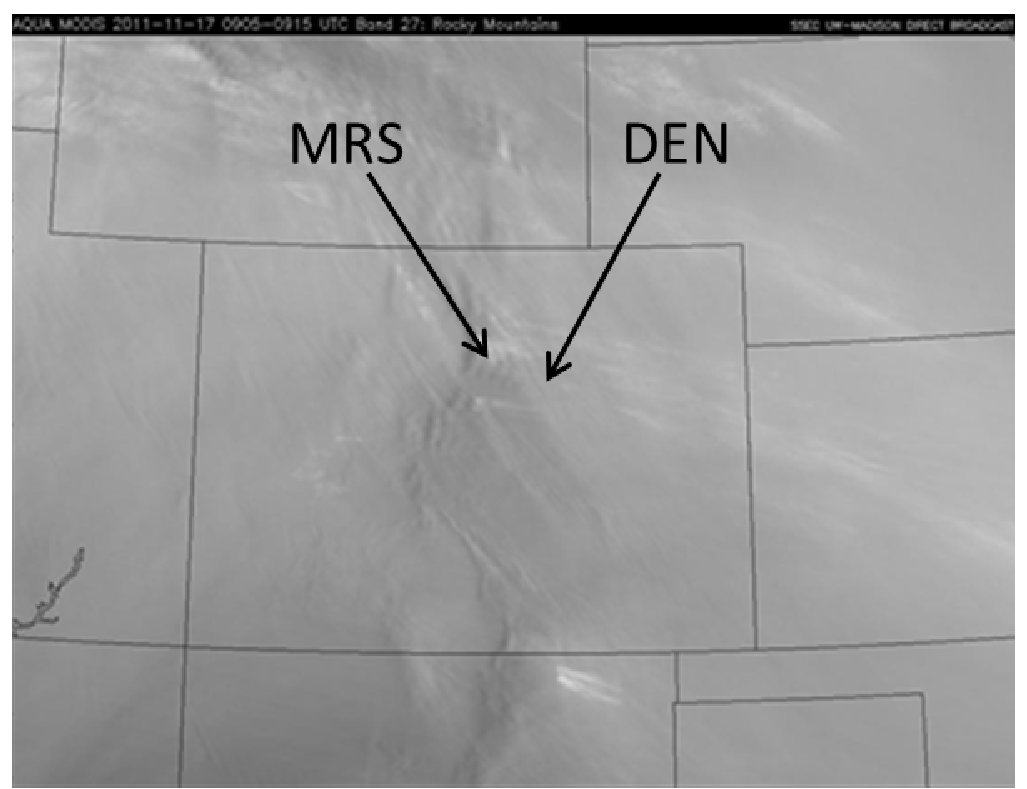

Figure 6. MODIS Imagery over Colorado Rockies, November 17, 2011. Band 27 (6.535 - 6.895 micron band) 


\section{Data Analysis}

Due to technical difficulties, some data sets collected at MRS in 2011 lacked not only webcam imagery to verify or exclude the existence of clouds, but also D\&P data. However, several runs from November 17, 2011 had complete data sets and lenticular clouds were present. Lenticular clouds are formed in a standing wave pattern if there is sufficient moisture in the atmosphere and are an indication of mountain wave activity. During the 4th time series from November 17, clouds were crossing the instrument field of view (FOV), which resulted in significantly larger disturbances of the measured signal than was observed in other data analyzed. This difference in disturbance is clearly seen in Figure 7, which shows spectral dependence for temporal variability of the signal in radiance units for all 8 runs processed to date. Figure 8 shows the same quantity in brightness temperature units for Nov. 17, run 4 only. The difference in disturbance is estimated by a calculation of temporal variability. This variability is estimated by performing statistics over all data cubes for a given run after subtracting the row mean radiance for each cube/channel separately to eliminate the radiance dependence on the viewing elevation angle.

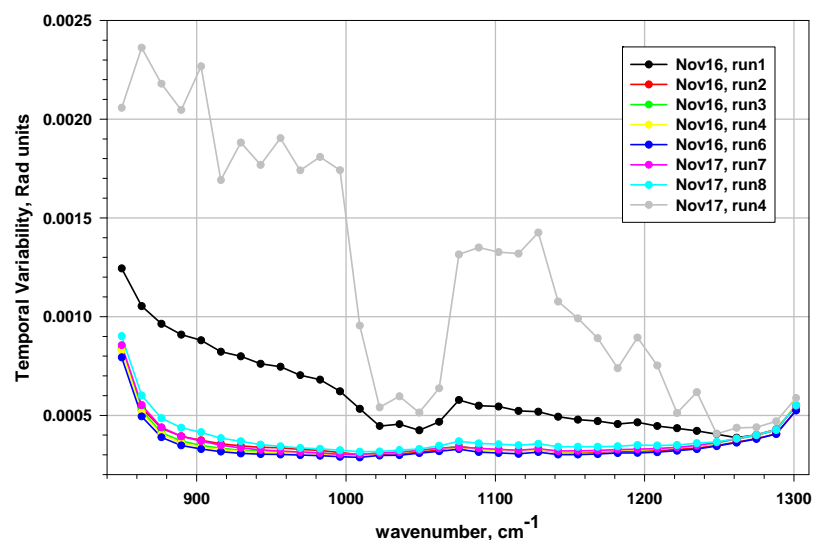

Figure 7.Spectral dependence of the temporal variability of the scene obtained by averaging over all image pixels for a given channel, 8 runs total, radiance units.

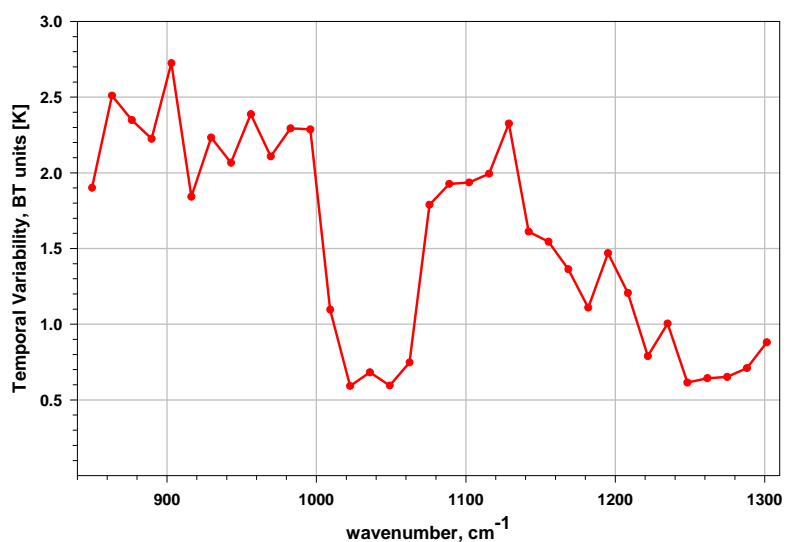

Figure 8. Spectral dependence of the temporal variability of the scene obtained by averaging over all image pixels for given channel, , brightness temperature units.

The computation of temporal variability produces one cube of data, which contains the estimated standard deviation for each pixel/channel separately. Averaging the standard deviation over all pixels, separately for each spectral channel, provides spectral dependence of the computed temporal variability. This result is presented in Figure 9 and Figure 10. Corresponding images of temporal variability for channels 4 and 23 are shown in Figure 9 and Figure 10. The most visible patterns in Figure 9 and Figure 10 are caused by cloud motion across the FOV during time series of measurements (see Figure 11). 
Temporal Variability

Nov 17, run 4, Channel $4\left(\sim 850 \mathrm{~cm}^{-1}\right)$

Rad. Scale $0-0.04 \mathrm{~W} / \mathrm{m}^{2} / \mathrm{cm}^{-1} / \mathrm{sr}$

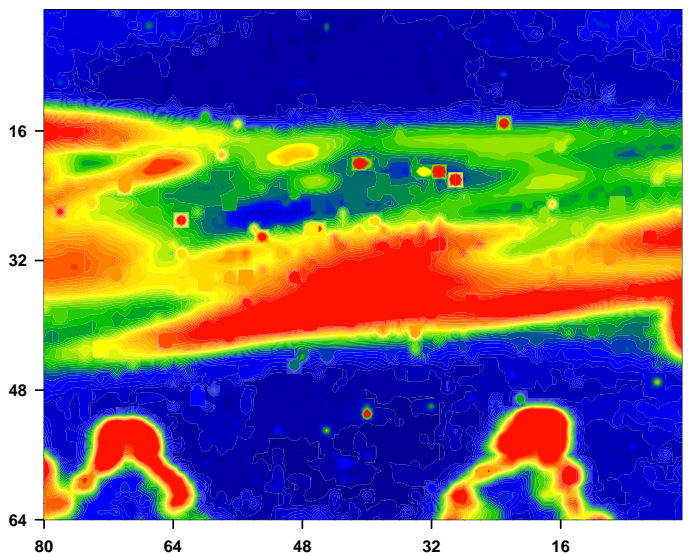

Temporal Variability

Nov 17, run 4, Channel $4\left(\sim 850 \mathrm{~cm}^{-1}\right)$

BrT. Scale 0-4 K

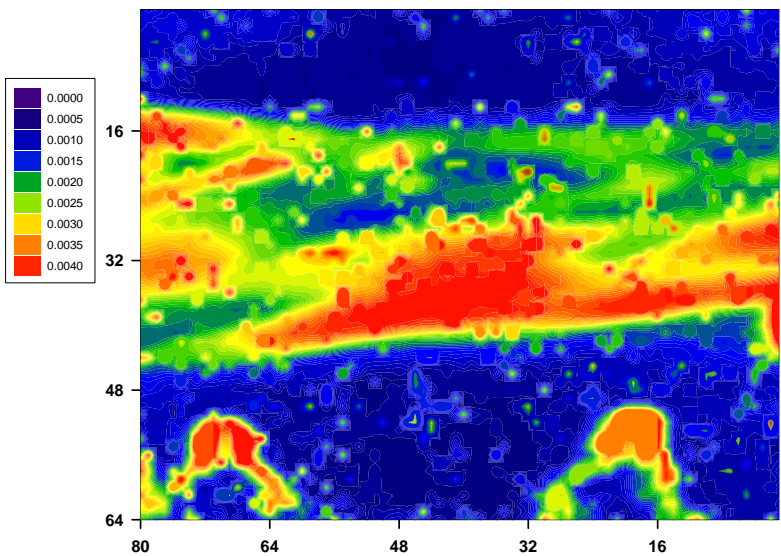

Figure 9. Image of temporal variability for channel 4, radiance units (left panel) and brightness temperature units (right panel): Nov 17, run 4.

Temporal Variability

Nov 17, run 4, Channel $23\left(\sim 1102 \mathrm{~cm}^{-1}\right)$ Rad. Scale 0-0.04 W/ $/ \mathrm{m}^{2} / \mathrm{cm}^{-1} / \mathrm{sr}$

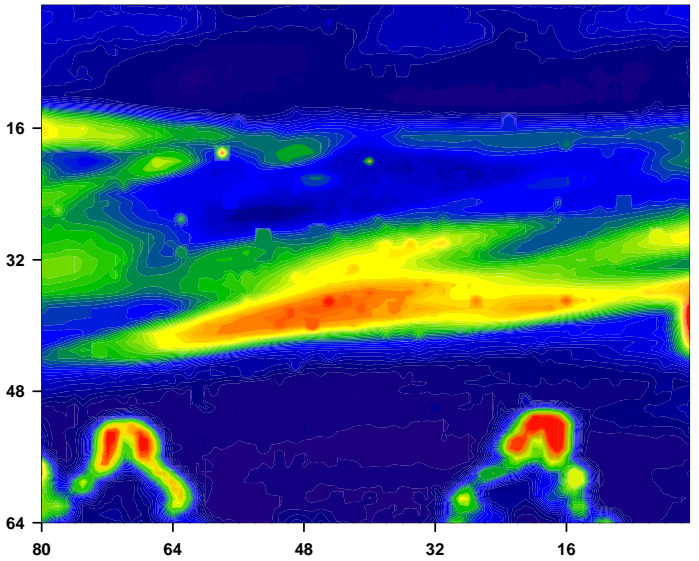

Temporal Variability

Nov 17, run 4, Channel $23\left(\sim 1102 \mathrm{~cm}^{-1}\right)$

BrT. Scale 0-4 K

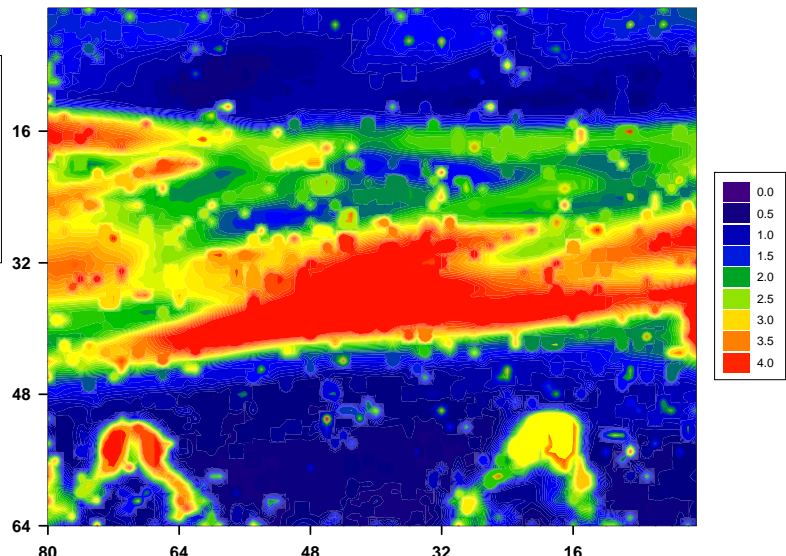

Figure 10. Image of temporal variability for channel 23, radiance units (left panel) and brightness temperature units (right panel): Nov 17 , run4.

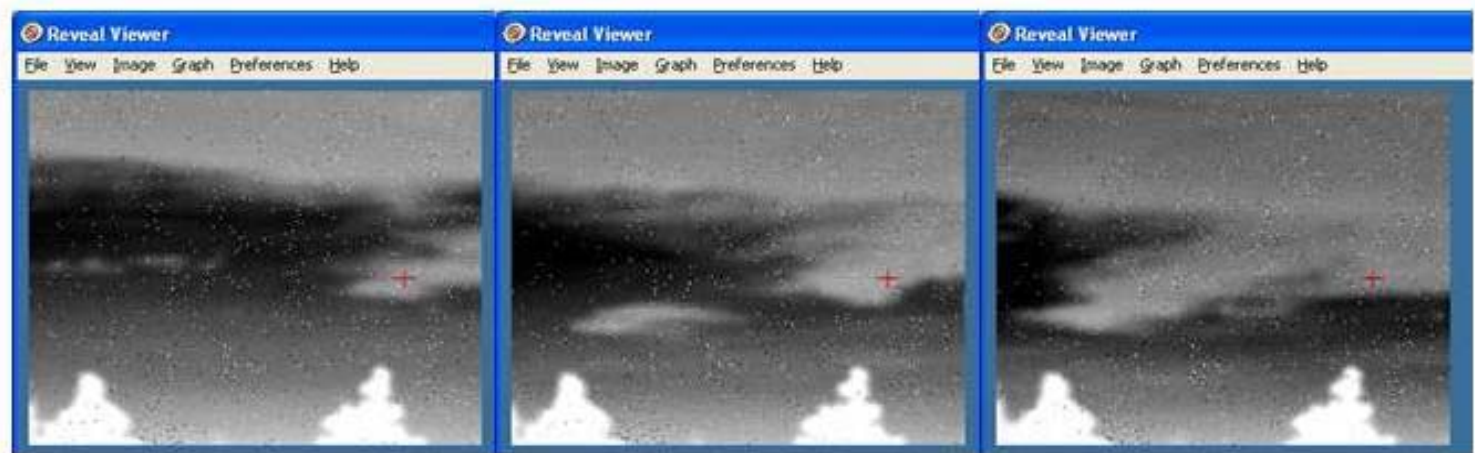

Figure 11. From left to right: channel \#5 from the first, middle, and last cubes of data, Nov 17, run 4. One can see how clouds are moving during time of the observation.

8

American Institute of Aeronautics and Astronautics 
In the above results, temporal variability had been computed over all good pixels, including cloudy ones. Now, we exclude them from the statistical averaging in order to reveal more clearly the temporal variability without cloud contamination. The cloud brightness temperature was estimated to be about $240 \mathrm{~K}$. We have performed computations for cloud brightness temperatures $\left(\mathrm{T}_{\mathrm{CLD}}\right)$ from $215 \mathrm{~K}$ to $250 \mathrm{~K}$ with $5 \mathrm{~K}$ increments to see how this threshold value affects the temporal variability pictures. A cloud mask was applied in such a way that all pixels with radiance $(\mathrm{R})$ values higher than $\mathrm{R}\left(\mathrm{T}_{\mathrm{CLD}}=215,250,5 \mathrm{~K}\right)$ have been excluded from the consideration. This exclusion leads to a different number of pixels averaged over 1007 data cubes total: for example, as can be seen in Figure 11, the upper and bottom thirds of the whole frame are always excluded, while for the central $1 / 3$ area, the number of averaged pixels is changing from 1007 (always clear) to 0 (always cloudy) from left to right. The number of averaged pixels depends also on the given value of threshold cloud temperature.

The temporal variability results, with cloud contributions over a threshold value $\left(\mathrm{T}_{\mathrm{CLD}}=240 \mathrm{~K}\right)$ being eliminated, are presented in Figure 12. Eight channels with central wavenumbers from $863 \mathrm{~cm}^{-1}$ to $1115 \mathrm{~cm}^{-1}$ are shown in brightness temperature units, with the same scale of 0 - $4 \mathrm{~K}$ for each panel.

The temporal variability when $\mathrm{T}_{\mathrm{CLD}}$ varies from $215 \mathrm{~K}$ to $250 \mathrm{~K}$ is presented in Figure 13 for the transparent channel at $\sim 903 \mathrm{~cm}^{-1}$. One can note that even as low as $215 \mathrm{~K}$, there is cloud signal. For comparison, Figure 14 shows temporal variability for the opaque channel at $\sim 1035 \mathrm{~cm}^{-1}\left(\mathrm{O}_{3}\right.$ band). The panels shown are for threshold values of $T_{C L D}=260 \mathrm{~K}, 265 \mathrm{~K}$, and $270 \mathrm{~K}$. There is no signal for $T_{C L D} \leq 255 \mathrm{~K}$ in this channel. Note also that the color scale is a factor of 2 smaller $(0-2 \mathrm{~K})$ in Figure 14 than in Figure 12 and Figure 13.
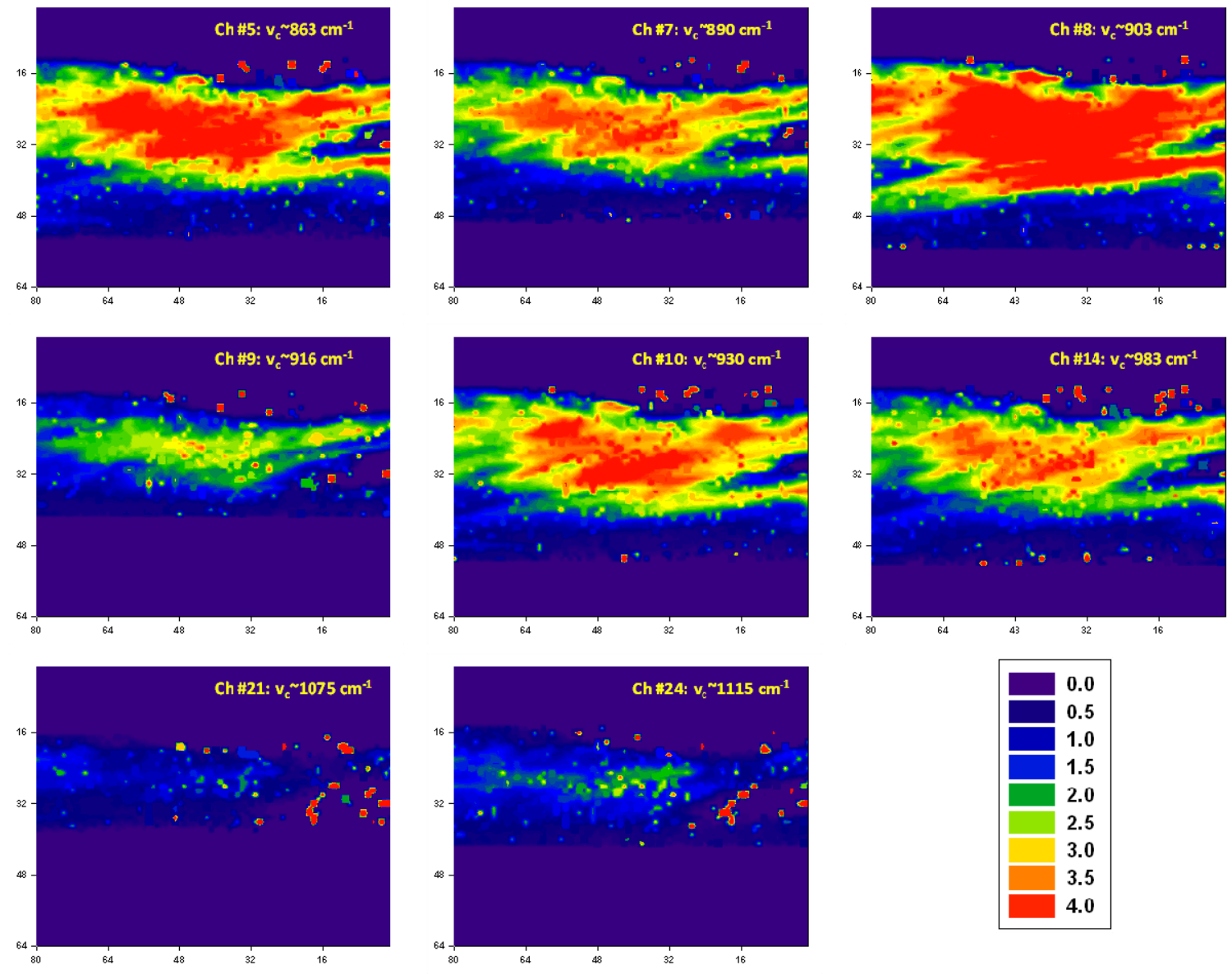

Figure 12. The temporal variability of the scene when cloudy pixels $\mathrm{TB}>\mathbf{2 4 0} \mathrm{K}$ are eliminated. Unit $=$ brightness temperature, the same color scale 0-4 K is used for all panels; Nov 17, run 4. 

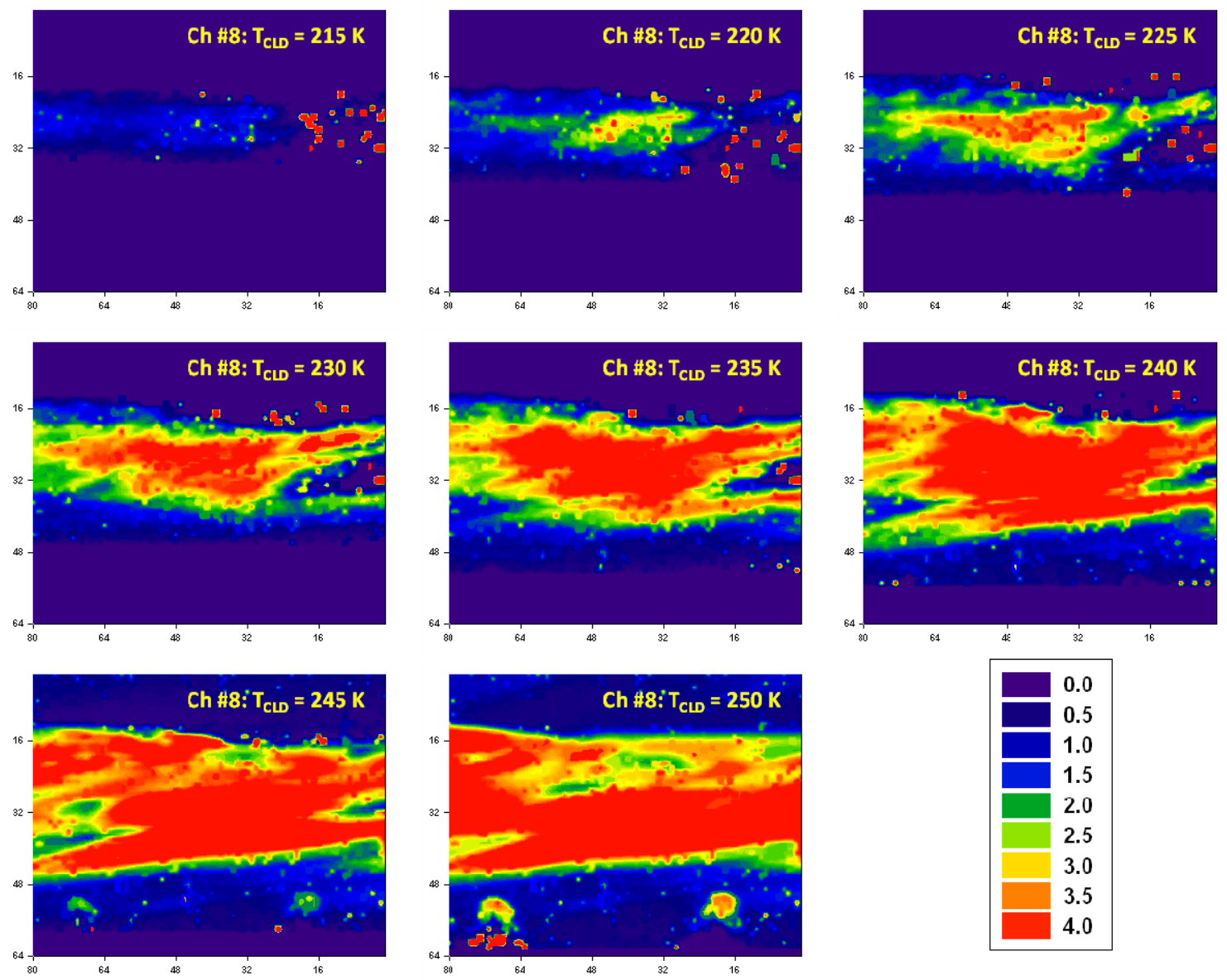

Figure 13. The temporal variability of the scene for the transparent channel $\# 8(\sim 903 \mathrm{~cm}-1)$ when different cloud brightness temperature threshold values are applied (215 K to $250 \mathrm{~K}$, increment $5 \mathrm{~K})$; Nov 17 , run 4.
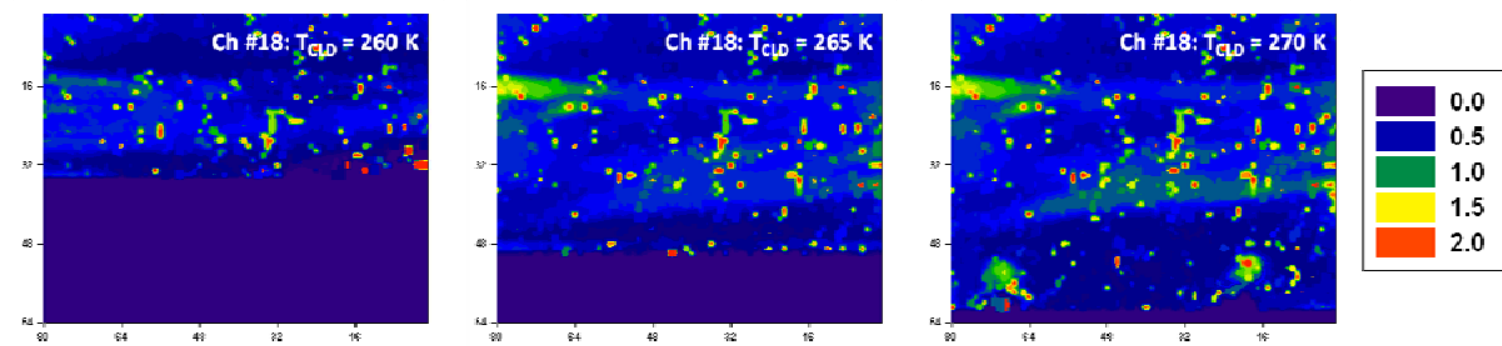

Figure 14. The temporal variability of the scene for the opaque channel \#18 $(\sim 1035 \mathrm{~cm}-1)$ when different cloud brightness temperature threshold values are applied $(260,265,270 \mathrm{~K})$; Nov 17 , run 4 . Note that color scale is a factor of two smaller $(0-2 \mathrm{~K})$ than the previous figures. There are no pixels for $\mathrm{T}_{\mathrm{CLD}} \leq 255 \mathrm{~K}$.

This study indicates that much of the variability seen is likely due to cloud variations, though more analysis is necessary. In a previous paper, we used different analysis techniques on the same data set. ${ }^{4}$ In this analysis, the sky at the horizon was not excluded from analysis. The cloud mask applied by Lane in [4] was based on spectral features of cloud versus sky, as opposed to temperature. A figure from this paper, which shows the high sky radiance at lower elevation, is shown in Figure 15. Even though the sky radiance is higher, there are still spectral features which differentiate it from clouds. 


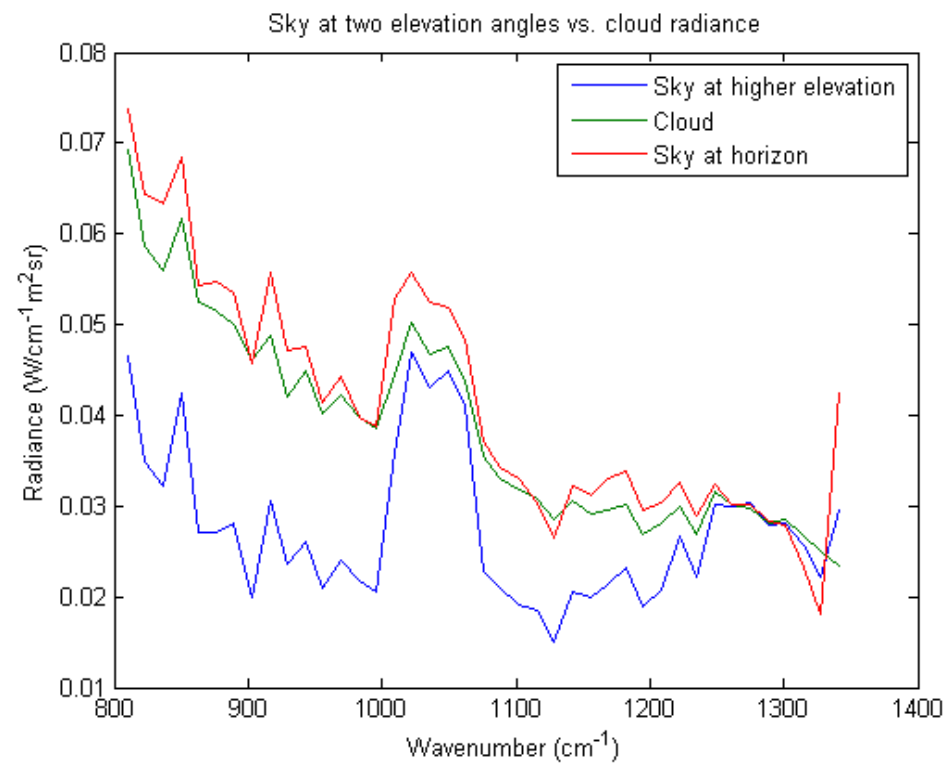

Figure 15. The radiance spectra from sky pixels at two elevations compared to the radiance of a cloud pixel. ${ }^{4}$

However, further analysis has shown that this portion of the sky is covered by a variable haze. Comparison of a truly clear day with the data from November $17^{\text {th }}$ shows the difference in the horizon. Figure 16 shows two LWIR images from the data collection activity at MRS. The left image is from November $16^{\text {th }}$, a completely clear day, while the image on the right is from November $17^{\text {th }}$. Note that the FOV's of the two images are not the same. For comparison, the green box in the left image outlines the approximate FOV of the right image. There is still a haze evident at the horizon. This haze is natural and expected, even on the clearest of days; the radiance will be higher toward the horizon and will decrease with increasing elevation angle. On the $17^{\text {th }}$, however, we believe that there is a thicker haze than is expected when compared with numbers from the clear day. The brightness temperatures for the clear day, shown at $903 \mathrm{~cm}^{-1}$ (the same channel as displayed for a cloudy day in Figure 13), are shown in Figure 17. On this clear day, approximate brightness temperature in the haze seems to indicate that $215 \mathrm{~K}$ could be a valid threshold for cloud masking. Further investigation and understanding is still warranted.
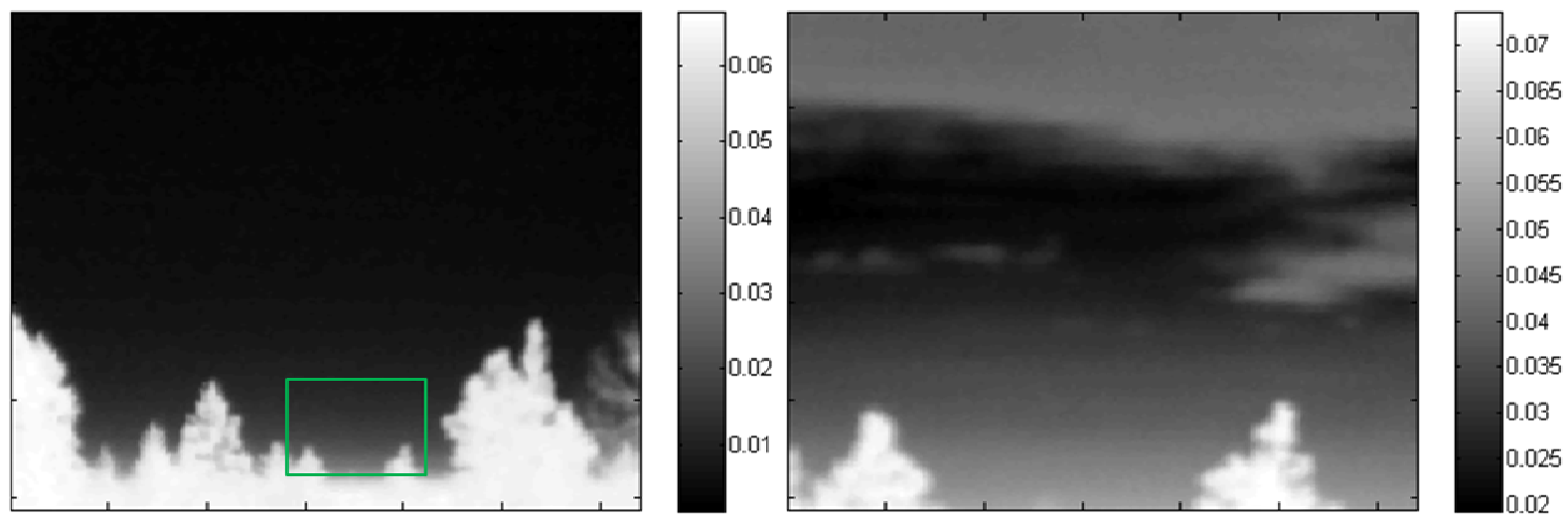

Figure 16.LWIR images from November 16, a clear day versus November 17, a cloudy day. Images shown are at $929.6 \mathrm{~cm}^{-1}$. The radiance is scaled in $\mathrm{W} / \mathrm{m}^{2} \mathrm{sr}$. 


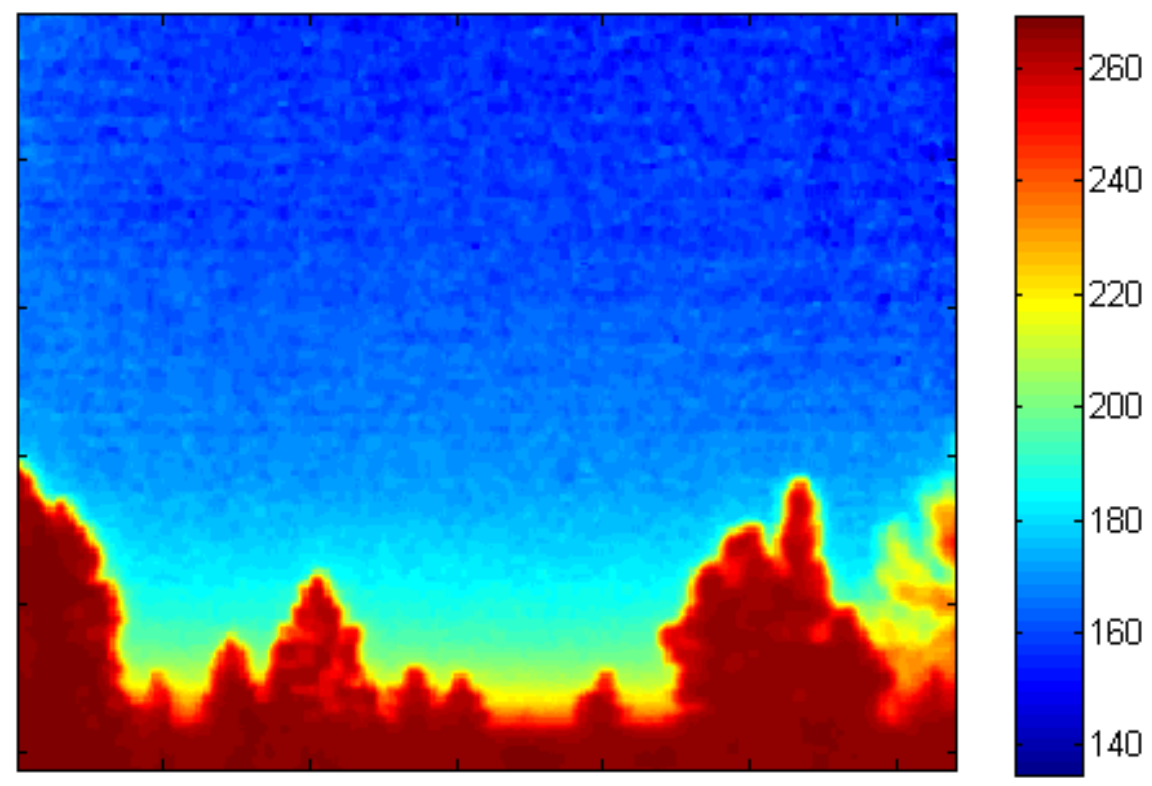

Figure 17. The brightness temperature of the sky on November 16, a clear day. Brightness temperature in Kelvin.

\section{Conclusion}

This paper presents a follow-on analysis to the SPIE paper ${ }^{4}$ referenced regarding the atmospheric data collected over the Colorado Rockies, near Boulder, CO. Brightness temperature differences were used in the identification of clouds, as opposed to spectral features of clouds versus sky. When the clouds are removed using the cloud brightness temperature threshold values, the variability is almost eliminated. In addition, further spectral analysis from the SPIE paper of clear sky versus haze along and above the horizon, an area that was masked by the cloud brightness temperature threshold used here, showed that the variability is likely due to hazy clouds that are not apparent in the visible spectrum.

Although these studies have not shown the expected results as seen in simulations, some of the expected results could be masked by the noise threshold of the data collection instrumentation. It may still be possible to see mountain waves with the Hyper-Cam; there is much remaining data to be analyzed. However, we are in the process of identifying an instrument with at least an order of magnitude reduction in measurement noise, relative to the Hyper-Cam. The Hyper-Cam sensitive range is 870 to 1300 wavenumbers. Research has shown sensitive regions for detection of atmospheric temperature variations in the lower troposphere are specific narrow bands: 670-750, 1250$1450,1580-1660,1800-2100$, and 2200-2350 wavenumbers. ${ }^{5}$ This could lead to the design of new instruments optimized for detection of atmospheric turbulence. Lastly, even if the Telops Hyper-Cam is unable to detect the expected signal of a mountain wave, we expect that the turbulence associated with mountain waves may still be evident in the data from the D\&P spectro-radiometer.

\section{Acknowledgments}

The NASA Aeronautics Research Mission Directorate (ARMD) Aviation Safety (AvSafe) Program, under the Atmospheric Hazard Sensing \& Mitigation (AHSM) area within the Atmospheric Environment Safety Technologies (AEST) project, funded the research described here. In addition, the authors would like to thank personnel from the Mountain Research Station for their guidance and assistance with the data collection activity presented here.

\section{References}

${ }^{1}$ Gimmestad, G. G., Papanicolopoulos, C. D., Richards, M. A., Sherman, D. L., and West, L. L.; "Feasibility Study of Radiometry for Airborne Detection of Aviation Hazards." Funded by the NASA Aviation Safety Program Intelligent Integrated Flight Deck Project, (NASA/CR-2001-210855 under contract NAS1-99073), June 2001.

${ }^{2}$ West, L .L., Gimmestad, G.G., Smith, W .L., Kireev, S., Cornman, L. B., Schaffner, P. R.; Tsoucalas, G.: Applications of a 
Forward-Looking Interferometer (FLI) for the On-board Detection of Aviation Weather Hazards. NASA Technical Publication TP-2008-215536, Oct. 2008

${ }^{3}$ West, L. L., Gimmestad, G.G., Smith, W.L., Kireev, S., Daniels, T., Cornman, L., Sharman, R., Weekley, A., Perram, G., Gross, K., Smith, G., Feltz, W., Taylor, J., and Olson, E., "Hazard Detection Analysis for a Forward-Looking Interferometer." Final Report NASA/ NNX07AN17A, Oct 31, 2008.

${ }^{4}$ Lane, S. E., West, L. L., Gimmestad, G.G., Kireev, S., Smith, W.L., Burdette, E. M., Daniels T. S., and Cornman. L., "Hyperspectral Image Turbulence Measurements of the Atmosphere," Proceedings of SPIE Infrared Imaging Systems: Design, Analysis, Modeling, and Testing XXIII, Vol. 8355, Baltimore, MD, 2012.

${ }^{5}$ Daniels, T. S., Smith, W. L., and Kirev, S.: 2012, "Recent Developments on Airborne Forward Looking Interferometer for the Detection of Wake Vortices", AIAA-1302175, 4th AIAA Atmospheric and Space Environments Conference, New Orleans, LA 